\title{
Internações por uso de substâncias psicoativas no estado do Ceará, Brasil
}

\section{Hospitalizations for use of psychoactive substances in the state of Ceará, Brazil}

\author{
Larissa Lauriano Sales ${ }^{1}$. Eugenio Moura Campos². Maximiliano Loiola Ponte de Souza ${ }^{3}$. \\ 1 Hospital Universitário Walter Cantídio (HUWC/UFC/EBSERH), Fortaleza, Ceará, Brasil. 2 Universidade Federal do Ceará \\ (UFC), Fortaleza, Ceará, Brasil. 3 Fundação Oswaldo Cruz (FIOCRUZ), Eusébio, Ceará, Brasil.
}

\section{RESUMO}

Objetivo: investigar os coeficientes de incidência de internações hospitalares por álcool e outras drogas (IHAD) no Ceará. Método: estudo descritivo utilizando dados do Sistema de Internações Hospitalares, do período 2013-2017. Resultados: o coeficiente de IHAD foi de 25,4/100 mil, sendo mais alta entre homens. Entre homens a substância isolada mais associada à internação foi o álcool, entre mulheres a cocaína igualou-se ao álcool. Os coeficientes de IHAD aumentaram conforme a idade nas três primeiras faixas etárias investigadas, caindo na última. Conclusão: foram identificadas variações de gênero e de faixa etária, assim como a importância do álcool enquanto substância isolada associada à internação.

Palavras-chave: Hospitalização. Transtornos relacionados ao uso de substâncias. Epidemiologia.

\section{ABSTRACT}

Objectives: to investigate the incidence coefficient of hospital admissions for alcohol and other drugs (IHAD) in Ceará. Method: Descriptive study using data from the Hospital Admissions System, from the period 2013-2017. Results: The coefficient of IHAD was 25.4/100 thousand, being higher among men. Among men, the single substance most associated with hospitalization was alcohol, among women, cocaine was equal to alcohol. IHAD coefficients increased with age in the first three age groups investigated, falling in the last. Conclusion: Gender and age variations were identified, as well as the importance of alcohol as an isolated substance associated with hospitalization.

Keywords: Hospitalization. Substance-related disorders. Epidemiology.

Autor correspondente: Maximiliano Loiola Ponte de Souza, Fundação Oswaldo Cruz (Fiocruz), Rua São José, sem número, Precabura, Eusébio, Ceará, Brasil. CEP: 61760-000. Telefone: +55 85 3215-6450. E-mail: maxkaelu@hotmail.com

Conflito de interesses: Não há qualquer conflito de interesses por parte de qualquer um dos autores.

Recebido em: 22 Mar 2021; Revisado em: 18 Jun 2021; Aceito em: 01 Set 2021. 


\section{INTRODUÇÃO}

Problemas relacionados ao uso de álcool e outras drogas são importantes agravos à saúde pública, acometendo de formas variáveis homens e mulheres, bem como as diferentes faixas etárias. $^{1}$ Tais agravos demandam abordagens terapêuticas complexas, em casos graves, requerendo internação hospitalar. Investigações sobre internações em contextos locais são importantes, pois podem fornecer informações simultaneamente a respeito dos casos graves que demandam assistência, assim como sobre a oferta de serviços hospitalares. Ainda que existam estudos sobre a temática, não se localizou estudos específicos realizados no estado do Ceará. Desta forma, o objetivo deste trabalho foi investigar os coeficientes de internações hospitalares por álcool e outras drogas (IHAD), e suas variações quanto a gênero e faixa etária no sistema público de saúde do Ceará.

\section{MÉTODO}

\section{Desenho do estudo}

Estudo descritivo, transversal e exploratório sobre a ocorrência de IHAD na população do estado Ceará, no período de 2013 a 2017.

\section{Caracterização do local da pesquisa}

O estado do Ceará está localizado na região nordeste do Brasil, e no período da pesquisa possuía uma população estimada em 8.452.381 habitantes, desigualmente distribuída entre as diferentes faixas etárias: $15,2 \%, 0-9$ anos; 32,7\%, 10 a 24 anos; 41,8\% 25 a 59 anos; 10,3\% (60 anos ou mais) (https:// sidra.ibge.gov.br/home/pmc/brasil).

\section{Fonte de dados}

Os dados sobre IHAD foram obtidos no Sistema de Informações Hospitalares (SIH) do Sistema Único de Saúde (SUS) (http://tabnet.datasus.gov.br/cgi/deftohtm.exe?sih/cnv/ nice.def). Os dados populacionais para o período investigado foram estimados a partir dos censos oficiais de 2000 e 2010 (https://sidra.ibge.gov.br/home/pmc/brasil), por meio de interpolação geométrica anual. Os dados foram extraídos no período de março de 2020.

\section{Critérios de inclusão}

Foram consideradas IHAD todas aquelas cujo diagnóstico principal foi classificado como "Transtornos mentais e comportamentais devidos ao uso de substância psicoativa", F10-F19.

\section{Mensurações e análises estatísticas}

A ocorrência de IHAD foi mensurada por meio de coeficientes de incidência obtidos pela razão entre número de internações e a respectiva população, sendo expressos em internações/100 mil habitantes. Foram calculados coeficientes considerando tanto a totalidade de IHAD, como os subgrupos diagnósticos de transtornos mentais e comportamentais devidos ao uso de substâncias (F10, álcool; F14, cocaína; F19, múltiplas drogas e outros), para população geral e para os diferentes sexos e faixas etárias. Foram estimados intervalos de confiança de 95\% (IC 95\%) para os diferentes coeficientes. Na análise considerou-se que havia diferença estatística entre os grupos quando não havia intersecções entre os intervalos de confiança de seus coeficientes.

\section{Aspectos éticos}

Foram utilizados exclusivamente dados secundários de livre acesso, de modo que o projeto do estudo foi dispensado de apreciação ética, em conformidade com a Resolução do Conselho Nacional de Saúde (CNS) no 510/2016.

\section{RESULTADOS}

Foram registradas 11.591 IHAD no Ceará no período investigado. O coeficiente de IHAD foi de 25,4/100 mil (25,0-25,9), 48,9/100 mil $(48,0-49,9)$ entre homens e 5,5/100 mil $(5,3-5,9)$, entre mulheres. Em todos os subgrupos diagnósticos e faixas etárias os coeficientes foram mais elevados entre homens. Em ambos os sexos, o coeficiente mais elevado foi encontrada no subgrupo F19. Entre homens, os coeficientes mais elevados foram dos subgrupos F10 (9,9/100 mil (19,3-20,5)) e F14 (6,6/100 mil $(6,3-7,0))$. Entre mulheres não se observou diferença estatística nas internações por estes grupos diagnósticos $(1,4 / 100$ mil $(1,2-$ 1,5) e 1,6/100 mil (1,5-1,8)) (Tabela 1).

Os coeficientes de IHAD em geral e para os diferentes subgrupos diagnósticos aumentaram nas três primeiras faixas etárias e caíram na última. Na população de 10-24 anos, de ambos os sexos, os coeficientes mais elevados foram encontrados no subgrupo F19, sendo de 12,1/100 mil (11,5-12,7), nesta faixa etária. Na faixa etária de 25-59 anos, entre homens, os coeficientes mais elevados ocorreram nos subgrupos F10 (39,5/100 mil (38,2-40,8)) e F19 (36,2/100mil $(34,9-37,4))$. Já entre mulheres, foram entre F19 (3,1/100 mil $(2,8-3,5))$ e F14 $(2,8 / 100$ mil $(2,5-3,1))$. No grupo de 60 anos ou mais, em ambos os sexos, o coeficiente mais elevado ocorreu no grupo F10, sendo de $6,4 / 100$ mil $(5,8-7,1)$ na faixa etária como um todo (Tabela 1).

\section{DISCUSSÃO}

O coeficiente de IHAD encontrado no Ceará $(25,4 / 100$ mil) foi mais baixo do que o observado em Alagoas (33,6/100 mil) e mais alto que o da Bahia (11,4/100 mil), estados da região nordeste com o maior e o menor coeficiente, respectivamente. ${ }^{2}$ Santa Catarina, na região sul, foi o estado do Brasil que apresentou, conforme a literatura, o mais elevado coeficiente, 132,6/100 mil. ${ }^{3}$ Mais do que as variações populacionais nos coeficientes de problemas relacionados ao uso substâncias nestes diversos contextos, é prudente propor, na medida em que não se observou diferenças metodológicas relevantes entre os estudos, que a importante diferença observada entre o coeficiente de Santa Catarina e os estados do nordeste possa estar associada a oferta de leitos, decorrente da disparidade socioeconômica e de acesso a serviços de saúde entre regiões do país. ${ }^{4}$ 
Tabela 1. Coeficientes de incidência* de internação psiquiátrica devido ao uso de álcool e outras drogas no estado do Ceará, por sexo e faixa etária. 2013-2017 ( $\mathrm{n}=11.591)$.

\begin{tabular}{|c|c|c|c|c|c|}
\hline Sexo & Idade & $\begin{array}{l}\mathrm{F} 10^{+} \\
\text {Coeficiente } \\
(\text { IC } 95 \%)^{\S \S}\end{array}$ & $\begin{array}{c}\mathrm{F} 14^{\S} \\
\text { Coeficiente } \\
\text { (IC 95\%) }\end{array}$ & $\begin{array}{c}\text { F19** } \\
\text { Coeficiente } \\
\text { (IC 95\%) }\end{array}$ & $\begin{array}{l}\text { F10-F19 } \\
\text { Coeficiente } \\
\text { (IC 95\%) }\end{array}$ \\
\hline \multirow[t]{5}{*}{ Masculino } & 0 a 9 anos & $0,0(0,0-0,2)$ & - & $0,1(0,0-0,3)$ & $0,5(0,3-0,8)$ \\
\hline & 10 a 24 anos & $2,0(1,7-2,4)$ & $3,8(3,3-4,3)$ & $20,8(19,7-22,0)$ & $27,3(26,0-28,6)$ \\
\hline & 25 a 59 anos & $39,5(38,2-40,8)$ & $12,4(11,7-13,2)$ & $36,2(34,9-37,4)$ & $88,9(87,0-90,9)$ \\
\hline & $\geq 60$ anos & $12,6(11,2-14,1)$ & $0,5(0,3-0,9)$ & $0,9(0,6-1,4)$ & $14,4(13,0-16,1)$ \\
\hline & $0 \mathrm{a} \geq 60$ anos & $19,9(19,3-20,5)$ & $6,6(6,3-7,0)$ & $21,7(21,1-22,4)$ & $48,9(48,0-49,9)$ \\
\hline \multirow[t]{5}{*}{ Feminino } & 0 a 9 anos & $0,1(0,0-0,3)$ & - & $0,0(0,0-0,2)$ & $0,4(0,3-0,7)$ \\
\hline & 10 a 24 anos & $0,3(0,2-0,5)$ & $1,3(1,1-1,6)$ & $3,3(2,9-3,8)$ & $5,2(4,7-5,8)$ \\
\hline & 25 a 59 anos & $2,4(2,1-2,7)$ & $2,8(2,5-3,1)$ & $3,1(2,8-3,5)$ & $8,7(8,2-9,3)$ \\
\hline & $\geq 60$ anos & $1,5(1,1-2,0)$ & $0,1(0,1-0,4)$ & $0,1(0,0-0,3)$ & $1,9(1,5-2,5)$ \\
\hline & $0 \mathrm{a} \geq 60$ anos & $1,4(1,2-1,5)$ & $1,6(1,5-1,8)$ & $2,3(2,1-2,5)$ & $5,5(5,3-5,9)$ \\
\hline \multirow[t]{5}{*}{ Ambos } & 0 a 9 anos & $0,0(0,0-0,2)$ & - & $0,0(0,0-0,2)$ & $0,5(0,3-0,7)$ \\
\hline & 10 a 24 anos & $1,2(1,0-1,4)$ & $2,5(2,3-2,8)$ & $12,1(11,5-12,7)$ & $16,3(15,6-17,0)$ \\
\hline & 25 a 59 anos & $19,2(18,6-19,8)$ & $7,2(6,8-7,5)$ & $18,1(17,5-18,7)$ & $45,0(44,1-45,9)$ \\
\hline & $\geq 60$ anos & $6,4(5,8-7,1)$ & $0,3(0,2-0,5)$ & $0,4(0,3-0,7)$ & $7,5(6,8-8,2)$ \\
\hline & $0 \mathrm{a} \geq 60$ anos & $9,8(9,5-10,1)$ & $3,9(3,7-4,1)$ & $11,3(11,0-11,6)$ & $25,4(25,0-25,9)$ \\
\hline
\end{tabular}

*Coeficiente de incidência= internações por 100 mil habitantes. $+\mathrm{F} 10=$ Transtornos mentais e comportamentais devidos ao uso de álcool; §F14= Transtornos mentais e comportamentais devidos ao uso da cocaína; **F19= Transtornos mentais e comportamentais devidos ao uso de múltiplas drogas e ao uso de outras substâncias psicoativas. ++F10-F19=Transtornos mentais e comportamentais devidos ao uso de substância psicoativa. $\S \S I C$ 95\% = Intervalo de confiança de $95 \%$.

Os coeficientes de internação foram maiores entre homens em todos os subgrupos diagnósticos, semelhante ao verificado na literatura. ${ }^{2,3}$ Os menores coeficientes de internação entre mulheres podem estar associados não só à menor prevalência populacional de uso de substâncias no sexo feminino, ${ }^{5}$ mas também à maior frequência de comportamento violento associado ao uso de substâncias entre homens, ${ }^{6}$ à menor oferta de leitos femininos ${ }^{3}$ e ao estigma de gênero que poderia resultar em menor procura por serviços de saúde por parte de mulheres. ${ }^{7}$ Apesar de menores, existem evidências que os coeficientes IHAD em mulheres vêm aumentando no Brasil. ${ }^{4}$ Os problemas relacionados ao uso de substâncias aumentam o risco para diversos agravos a saúde da mulher, ${ }^{7}$ sendo este grupo, a despeito dos menores coeficientes IHAD, um destinatário essencial das políticas públicas.

Em ambos os sexos, o mais alto o coeficiente foi encontrado no subgrupo F19. Tal achado foi semelhante ao observado nos estados do Piauí, Rio Grande do Norte, Paraíba e Sergipe. ${ }^{2}$ Por outro lado, internações devido ao uso de álcool (F10) foram as mais elevadas em mulheres no Maranhão e Bahia, ${ }^{2}$ e em ambos os sexos em Santa Catarina. ${ }^{8} \mathrm{O}$ uso de múltiplas substâncias é um fenômeno relativamente comum, na medida em que os usuários de uma substância tendem a usar outras com o objetivo de reduzir, aumentar ou modificar seu efeito. ${ }^{9}$
O uso de múltiplas substâncias consiste em fator de risco para recaídas e resultados insatisfatórios de tratamento, ${ }^{10} \mathrm{o}$ que complexifica a abordagem dos casos.

Entre homens, a substância isolada associada a maiores coeficientes de internação foi o álcool, e entre mulheres foram álcool e cocaína, com valores sem diferença estatística entre si. Diversos estudos ${ }^{11,12,13}$ apontam o álcool como principal substância isolada associada às internações psiquiátricas. A dependência ao álcool atinge cerca de $12 \%$ da população brasileira ${ }^{5}$ constituindo-se em um importante problema de saúde pública. Entretanto, as políticas e ações de saúde pública usualmente são direcionadas a não utilização das drogas ilícitas. ${ }^{11}$

Um aspecto importante encontrado nesta pesquisa foi a importância relativa da cocaína enquanto substância isolada na internação de mulheres. Tal achado não foi verificado em outros estados do Nordeste. ${ }^{2}$ Infelizmente, diversos outros estudos nacionais não apresentam dados a respeito de internações conforme substância específica entre mulheres. ${ }^{3,4,8,14}$ De todo modo, esta pesquisa aponta para a necessidade de maior atenção com os problemas relacionados ao uso de cocaína entre mulheres, algo que pode ser específico do contexto cearense, ou simplesmente não está sendo identificado em outros cenários. 
Observou-se um aumento progressivo dos coeficientes nos primeiros três intervalos etários investigados, seguido por uma queda no último. Tal achado é semelhante ao observado na literatura nacional. ${ }^{2,3} \mathrm{O}$ acesso a substâncias psicoativas é limitado na faixa etária infantil. As experimentações iniciamse em geral no início da adolescência, usualmente sendo necessário um tempo para que se avolume prejuízos pessoais, laborais e familiares que motivariam as internações nas faixas etárias seguintes. ${ }^{4,14}$ Os menores coeficientes encontrados em idosos poderiam estar associados a questões como: influência do uso problemático de substâncias na expectativa de vida, mudanças na prevalência de uso, no padrão de consumo ou da expressão de comportamentos amplamente disruptivos com a idade. Por outro lado, destaca-se que a despeito dos relativamente mais baixos coeficientes encontrados no Ceará, evidências nacionais ${ }^{4}$ e internacionais ${ }^{15}$ apontam para o aumento do mesmo ao longo dos anos. Destaca-se que o uso de substâncias pode afetar a saúde do idoso e piorar o prognóstico de comorbidades existentes. ${ }^{15}$

Foram encontradas ainda variações etárias nos coeficientes de internações por subgrupos específicos. Na faixa etária de 10-24 anos, independentemente do sexo, os coeficientes mais elevados ocorreram no subgrupo F19, podendo estar associada a maior experimentação nestes anos da vida. Entre homens de 25-59 anos, o coeficiente de internação por F19 iguala-se

\section{REFERÊNCIAS}

1. United Nations Office on Drugs and Crime. World Drug Report 2017. United Nations: Viena: United Nations publications; 2017.

2. Perez JA, Rios LA, Merelles SL, Duarte MB. Internações hospitalares por uso de substâncias psicoativas no nordeste brasileiro em 2018. Rev Ciênc Méd Biol. 2020;19(3):405-10.

3. Balbinot AD, Horta RL, Costa JSD, Araújo RB, Poletto S, Teixeira MB. Hospitalizações por uso de drogas não se alteram com uma década de Reforma Psiquiátrica. Rev Saúde Pública. 2016;50:26.

4. Rodrigues TF, Oliveira RR, Decesaro MN, Mathias TA. Aumento das internações por uso de drogas de abuso: destaque para mulheres e idosos. J. bras. psiquiatr. 2019;68(2):73-82.

5. Carlini EA, Galduróz JC, Noto AR, Carlini CM, Oliveira LG, Nappo AS, et al. II Levantamento domiciliar sobre o uso de drogas psicotrópicas no Brasil: estudo envolvendo as 108 maiores cidades do país - 2005. São Paulo (SP): UNIFESP, CEBRID; 2006.

6. Giusti JS, Sañudo A, Scivoletto S. Differences in the pattern of drug use between male and female adolescents in treatment. Rev Bras Psiquiatr 2002; 24(2): 80-2.

7. Blume SB. Chemical dependency in women. Important issues. Am J Drug Alcohol Abuse 1990; 16(3-4): 297-307.

8. Sakae T, Fernandez E, Magajewski F. Análise do perfil das internações hospitalares por drogadição em Santa Catarina entre a de F10, enquanto entre mulheres iguala-se ao de F14. Por fim, entre idosos, F10 foi o subgrupo com coeficientes mais elevados. Tais achados parecem apontar para a consolidação da importância dos problemas com o uso do álcool enquanto causa de internação com o aumento da idade, no contexto investigado, ainda que possa seguir padrões distintos entre homens e mulheres. A ampla aceitação social do consumo do álcool e os menores riscos para sua aquisição, em relação a outras substâncias podem estar associados a este achado.

Dentre as limitações deste trabalho destaca-se o fato de não incluir as internações realizadas em hospitais privados não conveniados ao SUS, nem aquelas realizadas em comunidades terapêuticas, nem separar diferentes internações de um mesmo paciente.

Adespeito das limitações, foi possível obter informações de mais de 11 mil IHAD, com completude de $100 \%$ para as variáveis investigadas. Foi possível identificar variações de gênero e de faixa etária, assim como a importância do álcool enquanto substância isolada relacionada à internação, e dos problemas relacionados ao uso de cocaína entre mulheres. Tais achados permitiram a construção de panorama da oferta e utilização de serviços públicos hospitalares na área de álcool e drogas no contexto do estado Ceará, podendo orientar adequações nos serviços e em políticas públicas no contexto local.

\section{8-2015. Arq Catarin Med. 2018;47(3):16-37}

9. Kondo A. The Characteristics of Law-evading Drug Users and Effective Approaches. Yakugaku Zasshi. 2016;136(1):89-94.

10. Fernandes M, Feitosa C, Mendes P, Figueiredo M, Oliveira A, Silva J. Hospitalizations due to psychoactive substances usage: a psychiatric hospital study. R Pesq.: Cuid. Fundam. 2020; 12: 1132-1138.

11. Cantarelli ND, Marchesan EK, Amaral MC, Lemos JC. Perfil dos usuários de substâncias psicoativas de um hospital universitário do Rio Grande do Sul. Revista Saúde (Santa Maria). 2014;40(01):85-90.

12. Sousa FSP, Oliveira EN. Caracterização das internações de dependentes químicos em Unidade de Internação Psiquiátrica do Hospital Geral. Ciênc Saúde Coletiva. 2010;15(3):671-677.

13. Lasser KE, Hanchate AD, McCormick D, Walley AY, Saitz R, Lin MY, Kressin NR. Massachusetts Health Reform's Effect on Hospitalizations with Substance Use Disorder-Related Diagnoses. Health Serv Res. 2018;53(3):1727-44.

14. Oliveira CA, Teixeira GM, Silva VP, Ferreira LS, Machado RM. Perfil epidemiológico das internações pelo uso/abuso de drogas na região centro-oeste de Minas Gerais. Enferm Foco. 2013;4(3,4):175-8.

15. Chhatre S, Cook R, Mallik E, Jayadevappa R. Trends in substance use admissions among older adults. BMC Health Services Research. 201;17:584 DOI 10.1186/s12913-017-2538-z.

\section{Como citar:}

Sales LL, Campos EM, Souza ML. Internações por uso de substâncias psicoativas no estado do Ceará, Brasil. Rev Med UFC. 2021;61(1):1-4. 